\section{LIE GROUPS}

Lectures on Lie Groups

By J. Frank Adams. (Mathematics Lecture Note Series.) Pp. xii + 182. (W. A. Benjamin: New York and Amsterdam, 1969.) n.p.

'THE theory of Lie groups occupies a central position in modern mathematics. This theory is of interest to workers in such fields as algebraic topology, differential geometry, algebra and functional analysis, in addition to its applications in theoretical physics. Although some good texts on the subject are available, the wide area from which its ideas are drawn make it more difficult to learn than more homogeneous branches of mathematics. This new text by Professor Adams in the Benjamin "Lecture Note Scries" will be very useful for mathematicians and research students wishing to acquaint themselves with the subject.

This book is not an exhaustive account, but aims to prove the main results on the representations of compact Lie groups, introducing those concepts which are necessary for this purpose. It is based on a course of lectures addressed primarily to algebraic topologists, but the book will also be useful to workers in other fields. It will be particularly useful as a textbook for a graduate course on Lie groups.

The author's style is concise, and this conciseness gives a pleasing clarity to the text. In some places, however, conciseness is carried to an extreme-particularly in chapter one where not all the definitions are given as clearly as one would wish.

After introducing the basic definitions, the author discusses 1-parameter subgroups and the exponential map. Properties of representation spaces are then discussed, including relations between real, complex and quaternionic representations, and the Peter-Weyl theorem is proved. Maximal tori in compact groups are then considered, and the usual concepts of roots, weights, the Weyl group, the Dynkin cliagram and the Stiefel diagram are introduced. The method for finding the fundamental group $\pi_{1}(G)$ from the Stiefel diagram is described. The connexion between the irreducible complex representations and the weights in the fundamental chamber is proved, and the structure of the representation ring of a compact connected simply connected group is derived. The proofs are complete except for some of the results in the early chapters, whore references are given instead. There are frequent examples in the text for the classical unitary, symplectic and orthogonal groups.

R. W. Carter

\section{SYNTHETIC ANTIBIOTICS}

Synthesis of Penicillin, Cephalosporin $\mathrm{C}$ and Analogs By Maghar S. Manhas and Ajay K. Bose. (New Directions in Organic Chemistry: A Series of Monographs based on Lectures and Seminars at Stevens Institute of Technology.) Pp. xii +118 . (Dekker: New York, October 1969.) $\$ 8.75$.

Two discoveries made at the turn of the last decade have led during the past ten years to the introduction of a wide range of $\beta$-lactam antibiotics into elinical medicine. The discovery by the Beccham Group that 6 -aminopenicillanic acid could be obtained by fermentation enabled thousands of semi-synthetic penicillins to be prepared. The second discovery made by Abraham and Newton was that cephalosporin $\mathrm{C}$ was a $\beta$-lactam antibiotic which was not destroyed by ponicillinases and was effective over a much wider antibacterial spectrum than the penicillins. In this book, Dr Manhas and Professor Bose review developments in this area, which have chiefly occurred during the past ten to fifteen years.

After a short introductory chapter, the use of physical methods is discussed. It is surprising, however, that while all the chief methods are presented, including recent applications of the nuclear Overhauser effect, the authors fail to mention the very interesting ultraviolet absorption spectra of the cephalosporins.

Chapters 3 and 4 constitute the major part of the book and are concerned with the synthesis of the penicillins and cephalosporins and their analogues respectively. These chapters provide a useful summary of the literature although the authors have perhaps given undue space to their own synthetic contributions. One of the most interesting features of cephalosporin chemistry has, however, been neglected. This concerns the unusual facility with which the allylic acetoxy-group is substituted by nitrogen and sulphur nucleophiles in aqueous medium. This type of substitution, first noticed by Abraham and Newton, has been extensively studied by the Glaxo group.

The mode of antibacterial action of the ponicillins is now very largely understood at the molecular level, largely because of the work of Strominger and his collaborators. It is surprising that this work is mentioned only very briefly and the conclusions regarding the essential molecular features for antibacterial activity are ignored. 'Those conclusions allow a rational approach for future synthetic endeavours which the authors anticipate.

The text contains very fow errors. Structural formulae are clearly presented and liberally used. The occasional omissions and mistakes in formulae are generally irritating rather than misleading. The literature is surveyed to July 1969.

G. Lowe

\section{LABORATORY TECHNIQUES}

\section{Electrophoresis of Proteins in Polyacrylamide and Starch Cells}

By A. H. Gordon. Pp. 149. Hfl.15; 35s.

An Introduction to Gel Chromatography

By L. Fischer. Pp. 246. Hfl.25; $58 s$.

Immunochemical Techniques for the Identification and Estimation of Macromolecules

By J. Clausen. Pp. 175. Hfl.15; 35s.

(Laboratory Techniques in Biochemistry and Molecular Biology, edited by T. S. Work and E. Work.) (North-Holland: Amsterdam and London, 1969.)

These books are paperback editions of the three parts which make up volume ono of a series "Laboratory Techniques in Biochemistry and Molecular Biology" edited by T. S. Work and E. Work. Thus the research worker can acquire an individual text on the tcchnique of most interest to him rather than purchase the complete volume which presumably will be principally destined for departmental libraries. All three rolumes set out to give expert practical advice and are intended for readers with little or no previous experience of the techniques discussed. This aim is satisfactorily achieved by all three authors, who adopt a similar approach: a brief outline of the basic principles followed by a detailed account of the methods, apparatus and practical applications.

The first book is devoted to gel electrophoresis using both polyacrylamide and starch supports. The author describes lucidly the advantages and disadvantages of both materials as applied to various problems. He discusses clearly the preparation of the gels, the choice of a suitable apparatus and the detection and recovery of separated material, and he provides a very useful appendix of buffer solutions.

The second book is an introduction to gel chromatography, and the author describes the preparation of the gel, the choice of a suitable column and the various elution techniques. The many practical applications mainly involve the use of 'Sephadex', but this is probably not due to any loyalties of the author to his employer, 